\title{
Evaluation of the acute and subchronic oral toxicity of ethanol extract from Valeriana pavoniispecies in Wistar rats*
}

\author{
María del Pilar Olaya, Qf, MSc ${ }^{1}$, María Constanza Lozano, MV, MSc ${ }^{2}$, \\ Lucía Botero, MV, MSc ${ }^{3}$, Javier Rincón, QF, PhD ${ }^{4}$, Mario Francisco Guerrero, MD, DSc ${ }^{5}$
}

\section{SUMMARY}

Introduction: One of the most frequent problems found in medicinal plants is the absence of clinical, toxicological, and pharmacological studies. Valeriana pavonii is one of the species used in Colombia as an anxiolytic. Further study of this specie is rendered to add information in the toxicological area.

Objective: The acute and subchronic oral toxicity of V. pavonii ethanolic extract was evaluated in Wistar rats of both sexes.

Materials and methods: The rats were distributed into four groups: the control group received the vehicle $(0.5 \mathrm{~mL} / 100$ $\mathrm{g}$ of corporal weight) and the other three groups received increasing levels of the dosage for 90 days to evaluate characteristics like physical exam, laboratory test (blood chemistry and haematology), and anatomopathological findings.

Results: This study reveals that there were no signs of toxicity, mortality, or significant alterations attributable to the ethanolic extract of $V$. pavonii.

Conclusions: The Not Observed Adverse Effect Levels (NOAEL) of V. pavonii ethanolic extract were $2000 \mathrm{and} 1000 \mathrm{mg} /$ $\mathrm{kg}$ of body weight for the acute and subchronic toxicity studies, respectively.

Keywords: Toxicity tests; LOAEL; Valerian; NOAEL; Anxiolytic; Medicinal plants.

\section{Evaluación de la toxicidad oral aguda y sub-crónica del extracto etanólico de la especie Valeriana pavonii en ratas} Wistar

\section{RESUMEN}

Introducción: Uno de los problemas más frecuentes asociados con el uso de plantas medicinales es la ausencia de evidencias farmacológicas, toxicológicas y clínicas. Valeriana pavonii es una de las especies más utilizadas popularmente en Colombia con fines ansiolíticos. Es necesario avanzar en el estudio de esta especie para aportar información en el campo toxicológico.

Objetivos: Evaluar la toxicidad oral aguda y sub-crónica del extracto etanólico de V. pavonii en ratas Wistar de ambos sexos.

Materiales y métodos: En cada uno de los estudios se distribuyeron ratas en cuatro grupos; un grupo control que recibió únicamente vehículo $(0.5 \mathrm{ml} / 100 \mathrm{~g}$ de peso corporal $)$ y tres grupos correspondientes a niveles crecientes de dosis, así: para el estudio de toxicidad aguda se administraron en dosis única 20,200 y $2000 \mathrm{mg} / \mathrm{kg}$ con un período de observación de 14 días y para el de toxicidad sub-crónica, dosis diarias de 250,500 y $1000 \mathrm{mg} / \mathrm{kg}$ durante 90 días. Se evaluaron los parámetros de examen

* This project was funded by the Research Direction Bogotá campus, Research Vice-Presidency of the Universidad Nacional de Colombia. Materials and reagents provided by the Department of Pharmacy, Faculty of Science, Universidad Nacional de Colombia, Bogotá, DC, Colombia.

1. Professor, Department of Toxicology, Faculty of Medicine, Universidad Nacional de Colombia, Bogotá, DC, Colombia. e-mail:mpolayao@unal.edu.co

2. Professor, Departamento de Farmacia, Faculty of Science, Universidad Nacional de Colombia, Bogotá, DC, Colombia. e-mail:mclozanoa@unal.edu.co

3. Assistant Professor, Department of Animal Health Science, Faculty of Veterinary Medicine and Animal Breeding, Universidad Nacional de Colombia, Bogotá, DC, Colombia. e-mail: lboteroe@ unal.edu.co

4. Associate Professor, Department of Pharmacy, Faculty of Science, Universidad Nacional de Colombia, Bogotá, DC, Colombia.e-mail:jrinconv@unal.edu.co

5. Titular Professor, Department of Pharmacy, Faculty of Science, Universidad Nacional de Colombia, Bogotá, DC, Colombia. e-mail:mfguerrerop@unal.edu.co

Received for publication July 23, 2009 Accepted for publication May 7, 2010 
físico, los exámenes de laboratorio (química sanguínea y hematología) y el estudio anatomopatológico.

Resultados: No se presentaron signos de toxicidad, letalidad ni alteraciones significativas atribuibles al consumo del extracto etanólico de V. pavonii, según el examen físico, el examen anatomopatológico y el análisis de las pruebas de química sanguínea y hematología.

Conclusiones: Los valores de nivel sin efectos adversos observados (NOAEL) del extracto etanólico de $V$. pavonii, fueron 2000 y $1000 \mathrm{mg} / \mathrm{kg}$ de peso corporal para los estudios de toxicidad aguda y sub-crónica, respectivamente. No se encontraron valores de nivel más bajo de efecto adverso observado (LOAEL).

Palabras clave: Pruebas de toxicidad; LOAEL; Valeriana; NOAEL; Ansiolítico; Plantas medicinales.

The use of medicinal plants has rapidly expanded throughout the world ${ }^{1}$. Although there are no accurate data to assess the global use of medicinal plants, the World Health Organization (WHO) has estimated that over $80 \%$ of the world's population uses traditional medicine routinely as their source of primary health care. Frequently, traditional treatments involve the use of plant extracts or their active principles ${ }^{2}$. The quantity and quality of data on safety and efficacy in traditional medicine, including medicinal plants, are not enough to satisfy the necessary criteria to support their use in the world 3 .

One of the main problems found in the field of medicinal plants is the lack of pharmacological, toxicological and clinical evidence. In many cases, this practice is commonly associated with their traditional use ${ }^{4}$. Only a small fraction among thousands of medicinal plants used in the world has been rigorously tested in controlled studies; therefore, the evidence of toxicity risks is even lower ${ }^{5}$.

Valeriana is one of the genera of plants used as a sedative to treat anxiety. However, their species show important differences regarding their chemical composition V. pavonii is a species widely used in Colombia, which contains $0.44 \%$ of alkaloid metabolites, whereas V. officinalis contains less than $0.10 \%$ of these kinds of compounds ${ }^{6}$.

There is a large trade in species of the genus Valeriana, not only as phytotherapeutic product but also as fresh material in marketplaces. In Colombia, both species are sold interchangeably, although their morphological differences are notable ${ }^{7}$. V. pavonii, a native Colombian species approved for sedative purposes, has the same indications of $V$. officinalis ${ }^{8}$, which is one of the best known and studied European species. However, unlike the latter, $V$. pavonii lacks toxicological studies ${ }^{9}$. In this research, the acute and subchronic toxicity of the ethanol extract of $V$. pavonii was evaluated in Wistar rats for 90 days. Part of the acute toxicity study was presented at the XICongreso Nacional de Farmacología y Terapéutica.

\section{MATERIALS AND METHODS}

Ethanol extract. Fresh plant material was collected in San Antonio del Tequendama (Cundinamarca, Colombia), located at $2,500 \mathrm{~m}$ above sea level in the Cordillera Oriental (one of the three main Colombian mountain ranges) in October 2006. Two specimens were classified and deposited in the Colombian National Herbarium and were identified with the following codes: 495179 and 495756 . Aerial parts of the plants were dried in a forced-air oven at $40^{\circ} \mathrm{C}$ for 48 hours and pulverized in a disc mill. The powder obtained was subjected to percolation with $96 \%$ ethanol. The solvent was removed by rotary evaporation at reduced pressure with an average temperature of $40^{\circ} \mathrm{C}$. Finally, the material was dried at room temperature in a vacuum desiccator protected from light. The vehicle used for dilution of the ethanol extract was obtained from the mixture of $10 \%$ propylene glycol, $10 \%$ glycerol, $1 \%$ polysorbate- 80 and distilled water in sufficient quantity to make up $100 \%$.

Animals. Two groups of 60 Wistar rats per sex (seven to nine weeks old), weighing between 140-202 g (females) and 180-270 g (males), provided by the Department of Pharmacy, Faculty of Science, at Universidad Nacional were used for the two toxicity studies. The animals were kept at room temperature, $22 \pm 1{ }^{\circ} \mathrm{C}$, and 12-hour light-dark cycles were provided. Feed and water were given ad libitum.

Toxicity studies. This study was designed based on the recommendations by the World Health Organization (WHO). We took into account the guidelines for assessing the toxicity of herbal medicines presented in its «General guidelines for methodologies on research and evaluation of traditional medicine» because they are suitable for medicinal plant products. Recommendations were followed in terms of number and sex of animals used. 
Acute toxicity. Forty rats were used and divided randomly into four treatment groups of 10 animals each (five females and five males). The control group received the vehicle $(0.5 \mathrm{ml} / 100 \mathrm{~g}$ body weight $)$ and the other three groups corresponding to three dosage levels: 20, 200 , and $2000 \mathrm{mg} / \mathrm{kg}$. The extract or vehicle was administrated by oral gavage in a single dose.

Subchronic toxicity. Eighty rats were randomly assigned into four groups with 20 animals each (10 males and 10 females). The control group received the vehicle $(0.5 \mathrm{ml} / 100 \mathrm{~g}$ body weight $)$ and the other three groups received three dosage levels: 250, 500, and 1000 $\mathrm{mg} / \mathrm{kg}$. The extract or vehicle was administered daily by oral gavage during 90 days.

\section{Parameters evaluated in toxicity studies}

Physical examination. The rats were observed daily for two weeks throughout the acute toxicity study, and for 90 days in the sub-chronic toxicity study. Mortality was recorded and the presence, absence, increase, or decrease of the following signs were compared with the basal condition:

In the central nervous system (CNS): motor activity, ataxia, analgesia, anesthesia, righting reflex, corneal reflex, pineal reflex, paralysis of paw, grasping activity, alarm reaction, tremors, head twitches, and convulsions. In the eyes: enophthalmos, exophthalmos, ptosis, myosis, mydriasis, nystagmus, lacrimation, chromodacryorrhea, relaxation of the nictitating membrane, and corneal opacity. In skin appearance: paleness, cyanosis, and hyperemia. In general signs: salivation, piloerection, erection of the tail (Straub tail), diarrhea, priapism, dehydration (Robichaud test), dyspnea, and nasal discharge. And «other signs»: passivity, aggressiveness, fear, and respiratory rate ${ }^{10}$. Body weight was recorded at the beginning and weekly thereafter in both studies.

Laboratory tests. At the end of the acute and subchronic toxicity studies, all animals were anesthetized with ether and the blood was collected from the abdominal aorta. Blood samples were collected in two tubes (one with EDTA) for determination of clinical chemistry tests [blood urea nitrogen (BUN), creatinine, alanine aminotransferase (ALT), aspartate aminotransferase (AST), bilirubin, sodium, glucose, amylase), and hematology (erythrocyte count, total and differential leukocyte count, platelet count, hemoglobin, hematocrit, and sedimentation rate]. These samples were processed in a clinical laboratory certified in the Quality Manage- ment System (QMS) according to ISO 9001. The sacrifice of animals was performed under anesthesia by exsanguinations after puncture of the aorta.

Pathology. All animals were subjected to necropsy at the end of the toxicity studies, or earlier in case of death. For histopathology, samples were taken from the CNS, heart, lung, liver, kidney, spleen, pancreas, and gastrointestinal tract and preserved in $10 \%$ buffered formaldehyde. The tissues were processed by routine technique for hematoxylin-eosin and, in some cases to confirm the presence of iron pigment, Perl's stain was used. Macro and micro procedures were carried out at the Pathology Laboratory, Faculty of Veterinary Medicine, Universidad Nacional. The lesions observed were classified as mild, moderate, or severe.

Statistical analysis. Longitudinal data-analysis with dichotomous outcome variables (generalized estimating equations, GEE) were used for physical examination data in which positive cases were analyzed with respect to tota ${ }^{11}$. The blood parameters were processed by a factorial analysis of variance (ANOVA) followed by the Tukey test to determine the treatments responsible for the significant differences. When variance was not homogeneous, the Kruskal-Wallis test was applied. Analysis of the pathological data was accomplished by using Chi-square test in which categories such as normal, mild, moderate, and severe were defined. The programs: Excel ${ }^{\circ}$, SPSS $® 16$, and Stata ${ }^{\circledR} 9$ were used. The criteria for statistical significance was $p<0.05$ in all cases.

Ethical considerations. This study was evaluated and approved in a meeting held on March 23, 2006 (Act No. 05) by the Faculty of Medicine Ethics Committee, at Universidad Nacional in Bogotá, DC, Colombia.

\section{RESULTS}

\section{Acute toxicity}

Physical examination. It was observed that no deaths or signs of toxicity occurred during the assay. Signs of passivity (10 rats) and piloerection (eight rats) were reported. The results were expressed as number of positive observed cases (presence of the sign), taking into account that the total number of cases in each parameter was 560. Significant differences in passivity (in sex and time) and piloerection (in time) parameters were observed. Passivity was higher in males (26 males 
Table 1

Effect of V. pavonii ethanol extract on the weight of Wistar rats during the acute study

\begin{tabular}{|c|c|c|c|c|c|c|}
\hline \multirow{2}{*}{$\begin{array}{l}\text { Dose } \\
\text { (mg/kg) }\end{array}$} & \multicolumn{3}{|c|}{ Females } & \multicolumn{3}{|c|}{ Males } \\
\hline & Initial weight & $1^{\text {st }}$ week & $2^{\text {nd }}$ week & Initial weight & $1^{\text {st }}$ week & $2^{\text {nd }}$ week \\
\hline $\operatorname{High}(2000)$ & $183.9 \pm 9.8^{*}$ & $177.9 \pm 4.6$ & $191.8 \pm 6$ & $230.9 \pm 26.1$ & $217.2 \pm 32.6$ & $249.8 \pm 24.5$ \\
\hline Mean (200) & $181.8 \pm 18$ & $186.4 \pm 15.1$ & $195.7 \pm 10.3$ & $210.9 \pm 21.2$ & $219.3 \pm 20$ & $240.0 \pm 17.4$ \\
\hline Low (20) & $181.4 \pm 15$ & $185.1 \pm 15.4$ & $195.6 \pm 13.2$ & $239.6 \pm 15.6$ & $246.1 \pm 16.2$ & $262.6 \pm 15.4$ \\
\hline Control & $178.4 \pm 13.9$ & $183.4 \pm 15$ & $193.5 \pm 16.9$ & $220.9 \pm 6.7$ & $229.5 \pm 17.1$ & $258.6 \pm 12.3$ \\
\hline
\end{tabular}

${ }^{*} \bar{x}+\mathrm{ems}$

Table 2

Blood chemistry values in Wistar rats 14 days after administration in single oral dosage of $V$. pavonii ethanol extract

\begin{tabular}{|c|c|c|c|c|c|c|c|c|}
\hline \multirow[t]{2}{*}{ Parameters } & \multicolumn{4}{|c|}{ Females } & \multicolumn{2}{|c|}{ Males } & \multirow[b]{2}{*}{$\begin{array}{l}\text { High dose } \\
\text { (2000) }\end{array}$} & \multirow[b]{2}{*}{ Control } \\
\hline & $\begin{array}{l}\text { Low dose } \\
(20)^{1}\end{array}$ & $\begin{array}{l}\text { Mean dose } \\
\text { (200) }\end{array}$ & $\begin{array}{l}\text { High dose } \\
(2000)\end{array}$ & Control & $\begin{array}{l}\text { Low dose } \\
(20)^{\star}\end{array}$ & $\begin{array}{l}\text { Mean dose } \\
\text { (200) }\end{array}$ & & \\
\hline Glucose (mg/dl) & $201.8 \pm 6.2^{2}$ & $177.6 \pm 32.5$ & $177.8 \pm 34.8$ & $165.6 \pm 18.3$ & $131.8 \pm 20.3$ & $170.2 \pm 36.7$ & $195.4 \pm 34.9$ & $200.0 \pm 24.8$ \\
\hline Creatinine (mg/dl) & $0.88 \pm 0.18$ & $0.70 \pm 0.06$ & $0.68 \pm 0.04$ & $0.62 \pm 0.02$ & $0.62 \pm 0.18$ & $0.50 \pm 0.04$ & $0.80 \pm 0.08$ & $0.54 \pm 0.06$ \\
\hline BUN (mg/dl) & $37.9 \pm 3.5$ & $34.0 \pm 4.1$ & $33.7 \pm 1.5$ & $34.4 \pm 2.5$ & $23.3 \pm 3.6$ & $25.7 \pm 3.1$ & $30.5 \pm 3.2$ & $29.4 \pm 3.9$ \\
\hline ALT U/I & $44.8 \pm 7.9$ & $43.6 \pm 6.4$ & $61.0 \pm 4.7$ & $34.8 \pm 7.1$ & $36.2 \pm 8.5$ & $55.6 \pm 19.3$ & $43.0 \pm 12.3$ & $55.0 \pm 4.7$ \\
\hline AST U/I & $99.8 \pm 6.8$ & $118.6 \pm 20.0$ & $204.8 \pm 206^{3}$ & $128.8 \pm 11.1$ & $150.4 \pm 29.1$ & $182.2 \pm 24.9$ & $121.8 \pm 33.3$ & $145.0 \pm 35.3$ \\
\hline $\begin{array}{l}\text { Total bilirubin } \\
(\mathrm{mg} / \mathrm{dl})\end{array}$ & $0.92 \pm 0.06$ & $1.20 \pm 0.26$ & $1.62 \pm 0.42$ & $0.94 \pm 0.08$ & $0.96 \pm 0.13$ & $0.84 \pm 0.08$ & $1.48 \pm 0.20$ & $0.98 \pm 0.10$ \\
\hline $\begin{array}{l}\text { Direct bilirubin } \\
(\mathrm{mg} / \mathrm{dl})\end{array}$ & $0.50 \pm 0.05$ & $0.58 \pm 0.07$ & $0.96 \pm 0.22^{2}$ & $0.42 \pm 0.07$ & $0.42 \pm 0.10$ & $0.40 \pm 0.06$ & $0.73 \pm 0.13^{2}$ & $0.42 \pm 0.08$ \\
\hline Sodium (mEq/l) & $135.1 \pm 3.9$ & $138.1 \pm 6.8$ & $110.8 \pm 3.8^{2}$ & $135.7 \pm 4.3$ & $131.4 \pm 3.2$ & $140.1 \pm 4.2$ & $139.8 \pm 7.3$ & $135.6 \pm 5.3$ \\
\hline Amylase (U/I) & $689.6 \pm 113.9$ & $612.0 \pm 85.2$ & $721.0 \pm 144.8$ & $497.6 \pm 124.3$ & $578.3 \pm 213.1$ & $627.7 \pm 232.0$ & $792.0 \pm 88.57$ & $744.5 \pm 131.1$ \\
\hline
\end{tabular}

$\begin{array}{lll}\text { 1. } \mathrm{mg} / \mathrm{kg} & \text { 2. } \bar{x} \pm \text { ems } & \text { 3. Significant difference with respect control group }(p<0.05)\end{array}$

and one female) during the two first days, while piloerection appeared during the last three days. No significant differences were observed in body-weight gain between treated and control groups (Table 1).

Clinical chemistry. Female rats in the highest dosage group showed significant differences of serum sodium (decreased) and AST (increased) levels. The direct bilirubin increased significantly in the highest dosage group in both females and males (Table 2).

Hematology. There were no significant differences in hematological parameters between treated and control groups (Table 3 ).

Pathology. There were no significant macroscopic and microscopic changes in any of the treatment groups (Figures 1a and 1b).

\section{Subchronic toxicity}

Physical examination. Significant differences between the treated and control groups were observed in the following parameters: passivity (42), piloerection (8), loss of alarm reaction (11), aggressiveness (16), nasal discharge (21), dyspnea (18), and fear (25) in a total of 80 rats.

Passivity showed significant differences in time, dosage, and sex. This parameter was higher in the high dosage group of males and during the last two weeks. Significant differences were observed in time and dosage 
Table 3

Hematology values in Wistar rats 14 days after administration in single oral dosage of $V$. pavonii ethanol extract

\begin{tabular}{|c|c|c|c|c|c|c|c|c|}
\hline \multirow[b]{2}{*}{ Parameters } & \multicolumn{4}{|c|}{ Females } & \multicolumn{4}{|c|}{ Males } \\
\hline & $\begin{array}{l}\text { Low dose } \\
(20)^{1}\end{array}$ & $\begin{array}{l}\text { Mean dose } \\
(200)\end{array}$ & $\begin{array}{l}\text { High dose } \\
(2000)\end{array}$ & $\overline{\text { Control }}$ & $\begin{array}{l}\text { Low dose } \\
(20)^{\star}\end{array}$ & $\begin{array}{c}\text { Mean dose } \\
(200)\end{array}$ & $\begin{array}{l}\text { High dose } \\
(2000)\end{array}$ & Control \\
\hline $\begin{array}{l}\text { Leukocyte } \\
\text { (thousands } / \mathrm{mm}^{3} \text { ) }\end{array}$ & $4.7 \pm 0.5^{*}$ & $6.0 \pm 0.7$ & $4.0 \pm 0.4$ & $5.2 \pm 0.2$ & $5.0 \pm 0.6$ & $6.3 \pm 0.8$ & $5.3 \pm 0.6$ & $8.5 \pm 0.9$ \\
\hline $\begin{array}{l}\text { Erythrocyte } \\
\left.\text { (million } / \mathrm{mm}^{3}\right)\end{array}$ & $6.79 \pm 0.16$ & $6.20 \pm 0.62$ & $5.97 \pm 0.47$ & $6.80 \pm 0.21$ & $7.07 \pm 0.27$ & $7.15 \pm 0.29$ & $6.81 \pm 0.42$ & $6.24 \pm 0.92$ \\
\hline Hemoglobin (g\%) & $14.7 \pm 0.3$ & $13.6 \pm 1.0$ & $13.4 \pm 0.3$ & $14.6 \pm 0.3$ & $15.0 \pm 0.6$ & $15.3 \pm 0.5$ & $14.8 \pm 0.8$ & $13.4 \pm 2.0$ \\
\hline Hematocrit (\%) & $41.5 \pm 1.1$ & $37.4 \pm 3.6$ & $35.2 \pm 2.8$ & $40.7 \pm 1.1$ & $42.3 \pm 2.0$ & $43.1 \pm 1.6$ & $41.2 \pm 2.7$ & $38.1 \pm 5.6$ \\
\hline $\begin{array}{l}\text { Platelet count } \\
\text { (thousands } / \mathrm{mm}^{3} \text { ) }\end{array}$ & $355 \pm 134$ & $295 \pm 104$ & $557 \pm 168$ & $389 \pm 100$ & $555 \pm 123$ & $436 \pm 119$ & $388 \pm 51$ & $277 \pm 67$ \\
\hline $\begin{array}{l}\text { Eritrosedimentation } \\
\text { rate }(\mathrm{mm} / 1 \mathrm{~h})\end{array}$ & $0.2 \pm 0.2$ & $0.0 \pm 0.0$ & $0.0 \pm 0.0$ & $0.0 \pm 0.0$ & $0.0 \pm 0.0$ & $0.0 \pm 0.0$ & $0.0 \pm 0.0$ & $0.0 \pm 0.0$ \\
\hline \multicolumn{9}{|l|}{ Differential leukocyte } \\
\hline Lymphocyte (\%) & $94.1 \pm 1.6$ & $89.3 \pm 2.8$ & $91.6 \pm 2.1$ & $91.8 \pm 1.8$ & $89.7 \pm 3.2$ & $91.0 \pm 3.0$ & $86.3 \pm 3.1$ & $91.6 \pm 2.1$ \\
\hline $\begin{array}{l}\text { Granulocyte } \\
\text { (neut, \%) }\end{array}$ & $3.7 \pm 1.7$ & $9.1 \pm 3.3$ & $4.6 \pm 2.2$ & $5.3 \pm 2.4$ & $8.9 \pm 3.9$ & $5.3 \pm 3.8$ & $11.7 \pm 4.1$ & $4.0 \pm 2.7$ \\
\hline
\end{tabular}

$-$

${ }^{*} x \pm \mathrm{ems}$

Table 4

Physical examination parameters that showed differences in gender or in dosage during subchronic toxicity study of $V$. pavonii ethanol extract

\begin{tabular}{|c|c|c|c|c|c|c|c|c|}
\hline \multirow[t]{2}{*}{ Parameter } & \multirow{2}{*}{\multicolumn{2}{|c|}{ Total positive cases ${ }^{1}$}} & \multicolumn{4}{|c|}{ Dose (mg/kg) } & \multicolumn{2}{|c|}{ Sex } \\
\hline & & & Low 20 & Mean 200 & High 2000 & Control & Females & Males \\
\hline Passivity & & 144 & $21^{2}$ & $44^{2}$ & $76^{2}$ & 3 & $29^{3}$ & $115^{3}$ \\
\hline \multicolumn{2}{|c|}{ Loss of alarm reaction } & 20 & $9^{2}$ & 5 & 4 & 2 & 12 & 8 \\
\hline \multicolumn{2}{|c|}{ Aggressiveness } & 21 & $4^{2}$ & $2^{2}$ & $2^{2}$ & 13 & 7 & 14 \\
\hline \multicolumn{2}{|c|}{ Nasal discharge } & 27 & 4 & 5 & $15^{2}$ & 3 & 10 & 17 \\
\hline \multicolumn{2}{|l|}{ Dyspnea } & 35 & 2 & 16 & 17 & 0 & $11^{3}$ & $24^{3}$ \\
\hline
\end{tabular}

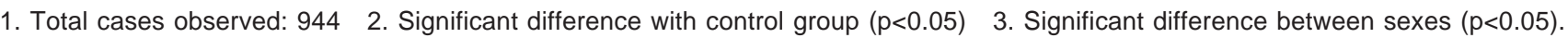

for loss of alarm reaction (increased during the study in the low dosage group). Aggressiveness was higher in the control group and between the second and seventh weeks. Nasal discharge increased in the highest dosage group and from the fifth week until the end of the study. Dyspnea presented differences in time and sex. This parameter was higher during the last two weeks and in males. Significant differences were observed throughout time for piloerection (during the first three weeks) and fear (higher during the first four weeks) (Table 4). No significant differences in body weight gain were observed between the treated and control groups (Table 5).

Clinical chemistry. Significant differences between females and males were observed for blood urea nitrogen (higher in females), glucose (higher in males) and direct bilirubin (lower in males) (Table 6).

Hematology. The erythrocyte count was significantly reduced in the high dosage group of females and males. There were no differences in other hematological parameters (Table 7). 
Table 5

Effect of $V$. pavonii ethanol extract on the weight of Wistar rats during the subchronic study

\begin{tabular}{|c|c|c|c|c|c|c|c|c|}
\hline \multirow[t]{2}{*}{ Week } & \multicolumn{4}{|c|}{ Males (dose mg/kg) } & \multicolumn{4}{|c|}{ Females (dose $\mathrm{mg} / \mathrm{kg}$ ) } \\
\hline & High dose 1000 & Mean dose 500 & Low dose 250 & Control & High dose 1000 & Mean dose & 500 Low dose 250 & Control \\
\hline 0 & $231.7 \pm 13.1^{*}$ & $226.3 \pm 6.2$ & $223.3 \pm 10.3$ & $225.9 \pm 8.4$ & $174.7 \pm 6.2$ & $178.2 \pm 5.5$ & $179.2 \pm 5.3$ & $174.6 \pm 6.6$ \\
\hline 1 & $237.1 \pm 16.5$ & $231.3 \pm 8.7$ & $234.9 \pm 11.2$ & $236.8 \pm 9.4$ & $176.8 \pm 6.0$ & $183.4 \pm 5.8$ & $182.1 \pm 4.5$ & $177.6 \pm 6.3$ \\
\hline 2 & $241.6 \pm 16.1$ & $232.5 \pm 8.3$ & $241.7 \pm 10.7$ & $246.6 \pm 9.3$ & $185.3 \pm 4.7$ & $191.1 \pm 6.6$ & $190.3 \pm 5.4$ & $189.5 \pm 5.7$ \\
\hline 3 & $256.8 \pm 19.9$ & $242.2 \pm 9.7$ & $254.4 \pm 11.1$ & $260.0 \pm 10.4$ & $189.7 \pm 3.8$ & $200.3 \pm 8.4$ & $198.1 \pm 4.4$ & $194.9 \pm 6.7$ \\
\hline 4 & $268.1 \pm 16.1$ & $257.0 \pm 9.8$ & $270.7 \pm 10.8$ & $272.3 \pm 11.1$ & $194.4 \pm 3.5$ & $207.4 \pm 8.2$ & $202.0 \pm 4.1$ & $203.3 \pm 5.3$ \\
\hline 5 & $276.7 \pm 15.1$ & $262.4 \pm 9.1$ & $275.7 \pm 10.3$ & $284.6 \pm 13.7$ & $199.8 \pm 4.4$ & $212.0 \pm 7.2$ & $205.7 \pm 5.0$ & $208.4 \pm 4.9$ \\
\hline 6 & $287.2 \pm 14.9$ & $272.2 \pm 9.2$ & $285.9 \pm 10.3$ & $285.4 \pm 10.8$ & $202.4 \pm 5.0$ & $216.6 \pm 7.8$ & $209.5 \pm 4.9$ & $206.5 \pm 7.3$ \\
\hline 7 & $293.6 \pm 15.2$ & $279.8 \pm 8.7$ & $293.2 \pm 10.3$ & $290.1 \pm 10.3$ & $203.3 \pm 5.2$ & $221.6 \pm 9.2$ & $215.1 \pm 6.3$ & $207.7 \pm 7.2$ \\
\hline 8 & $304.1 \pm 16.1$ & $290.5 \pm 9.3$ & $301.8 \pm 11.6$ & $297.7 \pm 9.9$ & $207.0 \pm 5.0$ & $228.6 \pm 9.6$ & $219.1 \pm 6.4$ & $211.9 \pm 7.5$ \\
\hline 9 & $313.7 \pm 17.1$ & $296.2 \pm 9.3$ & $309.7 \pm 12.0$ & $305.4 \pm 10.0$ & $208.1 \pm 5.0$ & $231.0 \pm 10.1$ & $221.2 \pm 6.4$ & $214.8 \pm 7.3$ \\
\hline 10 & $320.2 \pm 17.2$ & $300.4 \pm 9.5$ & $313.0 \pm 11.9$ & $312.6 \pm 9.8$ & $211.1 \pm 4.9$ & $233.8 \pm 10.1$ & $223.1 \pm 6.9$ & $217.1 \pm 7.6$ \\
\hline 11 & $327.9 \pm 18.5$ & $305.6 \pm 9.7$ & $319.3 \pm 13.1$ & $322.8 \pm 10.1$ & $213.8 \pm 4.9$ & $236.8 \pm 10.0$ & $226.3 \pm 7.0$ & $219.2 \pm 7.9$ \\
\hline 12 & $330.3 \pm 16.9$ & $308.7 \pm 9.6$ & $325.8 \pm 14.1$ & $327.5 \pm 10.4$ & $212.7 \pm 5.2$ & $236.7 \pm 11.2$ & $224.7 \pm 6.9$ & $219.0 \pm 8.0$ \\
\hline 13 & $331.1 \pm 16.2$ & $314.3 \pm 10.5$ & $329.9 \pm 15.1$ & $329.4 \pm 10.8$ & $220.4 \pm 5.7$ & $236.2 \pm 12.2$ & $224.0 \pm 3.1$ & $216.3 \pm 6.2$ \\
\hline
\end{tabular}

$* \bar{x} \pm \mathrm{ems}$

Table 6

Clinical chemistry values in Wistar rats after oral administration of $V$. pavonii ethanol extract for 90 days

\begin{tabular}{|c|c|c|c|c|c|c|c|c|}
\hline \multirow[t]{2}{*}{ Parameters } & \multicolumn{4}{|c|}{ Females (dose $\mathrm{mg} / \mathrm{kg}$ ) } & \multicolumn{4}{|c|}{ Males (dose $\mathrm{mg} / \mathrm{kg}$ ) } \\
\hline & Low dose 250 & Mean dose 500 & High dose 1000 & 0 Control & Low dose 250 & Mean dose 50 & OHigh dose 1000 & 0 Control \\
\hline $\begin{array}{l}\text { Glucose } \\
\text { (mg/dl) }\end{array}$ & $107.1 \pm 6.6^{1,2}$ & $108.0 \pm 9.3^{1}$ & $100.5 \pm 6.4^{1}$ & $110.2 \pm 10.5^{1}$ & $163.8 \pm 11.9^{1}$ & $153.4 \pm 11.8^{1}$ & $138.6 \pm 14.1^{1}$ & $162.0 \pm 16.0^{1}$ \\
\hline $\begin{array}{l}\text { Creatinine } \\
(\mathrm{mg} / \mathrm{dl})\end{array}$ & $0.81 \pm 0.05$ & $0.87 \pm 0.08$ & $0.89 \pm 0.05$ & $0.79 \pm 0.07$ & $0.74 \pm 0.02$ & $0.72 \pm 0.03$ & $0.74 \pm 0.03$ & $0.81 \pm 0.08$ \\
\hline $\operatorname{BUN}(\mathrm{mg} / \mathrm{dl})$ & $24.6 \pm 2.0^{*}$ & $24.5 \pm 2.6^{1}$ & $25.0 \pm 1.9^{1}$ & $22.1 \pm 1.8^{1}$ & $18.2 \pm 1.1^{1}$ & $19.4 \pm 0.9^{1}$ & $20.4 \pm 1.0^{1}$ & $21.0 \pm 1.2^{1}$ \\
\hline ALT U/ & $34.3 \pm 3.7$ & $42.5 \pm 6.9$ & $28.8 \pm 3.4$ & $36.8 \pm 4.4$ & $46.1 \pm 8.1$ & $38.8 \pm 4.5$ & $33.0 \pm 2.1$ & $48.3 \pm 5.4$ \\
\hline AST U/I & $139.0 \pm 13.3$ & $207.9 \pm 28.8$ & $170.5 \pm 28.4$ & $148.9 \pm 12.7$ & $182.0 \pm 22.4$ & $182.8 \pm 15.4$ & $160.5 \pm 12.4 \quad 1$ & $185.7 \pm 20.0$ \\
\hline $\begin{array}{l}\text { Total bilirubin } \\
(\mathrm{mg} / \mathrm{dl})\end{array}$ & $0.91 \pm 0.22$ & $1.01 \pm 0.23$ & $1.03 \pm 0.12$ & $0.83 \pm 0.09$ & $0.73 \pm 0.07$ & $0.71 \pm 0.05$ & $0.59 \pm 0.04$ & $0.75 \pm 0.13$ \\
\hline $\begin{array}{l}\text { Direct bilirubin } \\
(\mathrm{mg} / \mathrm{dl})\end{array}$ & $0.51 \pm 0.17^{1}$ & $0.53 \pm 0.14^{1}$ & $0.52 \pm 0.08^{1}$ & $0.44 \pm 0.06^{1}$ & $0.27 \pm 0.07^{1}$ & $0.28 \pm 0.05^{1}$ & $0.21 \pm 0.04^{1}$ & $0.30 \pm 0.08^{1}$ \\
\hline $\begin{array}{l}\text { Indirect bilirubin } \\
(\mathrm{mg} / \mathrm{dl})\end{array}$ & $0.41 \pm 0.07$ & $0.48 \pm 0.09$ & $0.52 \pm 0.05$ & $0.39 \pm 0.05$ & $0.46 \pm 0.05$ & $0.43 \pm 0.04$ & $0.38 \pm 0.03$ & $0.45 \pm 0.07$ \\
\hline $\begin{array}{l}\text { Sodium } 139.0 \pm 1 \\
(\mathrm{mEq} / \mathrm{l})\end{array}$ & $1.5144 .1 \pm 2.9$ & $143.1 \pm 3.1$ & $145.5 \pm 2.2$ & $138.6 \pm 2.2$ & $139.0 \pm 1.2$ & $142.0 \pm 3.4$ & $139.8 \pm 1.6$ & \\
\hline $\begin{array}{l}\text { Amylase } \\
(\mathrm{U} / \mathrm{dl})\end{array}$ & $260.8 \pm 21.5$ & $222.0 \pm 15.3$ & $246.9 \pm 13.9$ & $240.7 \pm 14.4$ & $298.3 \pm 19.3$ & $322.0 \pm 34.5$ & $331.3 \pm 32.1$ & $323.8 \pm 34.9$ \\
\hline
\end{tabular}

$\begin{array}{ll}\text { 1. Significant difference between sexes }(p<0.05) & \text { 2. } \bar{x} \pm \text { ems }\end{array}$ 
Table 7

Hematology values in Wistar rats after oral administration of $V$. pavonii ethanol extract for 90 days

\begin{tabular}{|c|c|c|c|c|c|c|c|c|}
\hline Parameters & Low dose 250 & \multicolumn{3}{|c|}{ Females (dose mg/kg) } & \multicolumn{4}{|c|}{ Males (dose mg/kg) } \\
\hline $\begin{array}{l}\text { Leukocyte } \\
\text { (thousands } / \mathrm{mm}^{3} \text { ) }\end{array}$ & $4.7 \pm 0.6^{1}$ & $5.3 \pm 0.9$ & $4.5 \pm 0.3$ & $3.8 \pm 0.5$ & $5.6 \pm 0.6$ & $5.0 \pm 0.9$ & $5.1 \pm 0.3$ & $4.2 \pm 0.5$ \\
\hline $\begin{array}{l}\text { Erythrocyte } \\
\text { (million } / \mathrm{mm}^{3} \text { ) }\end{array}$ & $6.88 \pm 0.26$ & $6.79 \pm 0.21$ & $5.12 \pm 0.55^{2}$ & $6.37 \pm 0.42$ & $7.25 \pm 0.26$ & $7.41 \pm 0.21$ & $6.88 \pm 0.55^{2} \quad 8$ & $3.00 \pm 0.42$ \\
\hline $\begin{array}{l}\text { Hemoglobin } \\
(\mathrm{g} \%)\end{array}$ & $15.0 \pm 0.6$ & $14.9 \pm 0.5$ & $11.7 \pm 1.1$ & $13.7 \pm 0.8$ & $15.0 \pm 0.6$ & $15.1 \pm 0.5$ & $14.5 \pm 1.1$ & $14.9 \pm 0.8$ \\
\hline Hematocrit (\%) & $40.8 \pm 1.5$ & $40.6 \pm 1.3$ & $31.8 \pm 3.3$ & $35.4 \pm 3.2$ & $41.5 \pm 1.5$ & $42.3 \pm 1.3$ & $40.2 \pm 3.3$ & $42.0 \pm 3.2$ \\
\hline $\begin{array}{l}\text { Platelet count } \\
\text { (thousands } / \mathrm{mm}^{3} \text { ) }\end{array}$ & $950.0 \pm 155.9$ & $772.0 \pm 101.0$ & $869.0 \pm 102.2$ & $880.6 \pm 59.0$ & $731.5 \pm 155.9$ & $965.5 \pm 101.0$ & $922.0 \pm 102.28$ & $850.2 \pm 59$ \\
\hline $\begin{array}{l}\text { Eritrosedimen- } \\
\text { tation }(\mathrm{mm} / 1 \mathrm{~h})\end{array}$ & $0.0 \pm 0.0$ & $0.0 \pm 0.0$ & $0.0 \pm 0.0$ & $0.0 \pm 0.0$ & $0.0 \pm 0.0$ & $0.0 \pm 0.0$ & $0.0 \pm 0.00 .0 \pm$ & $=0.0$ rate \\
\hline \multicolumn{9}{|c|}{ Differential leukocyte } \\
\hline Lymphocyte \% & $90.8 \pm 2.2$ & $92.7 \pm 2.0$ & $93.4 \pm 1.1$ & $92.6 \pm 0.9$ & $92.5 \pm 2.2$ & $94.1 \pm 2.0$ & $90.0 \pm 1.1$ & $91.4 \pm 0.9$ \\
\hline $\begin{array}{l}\text { Granulocyte } \\
\text { (neut) } \%\end{array}$ & $5.4 \pm 2.7$ & $4.4 \pm 2.2$ & $1.9 \pm 0.7$ & $4.1 \pm 1.2$ & $3.0 \pm 2.7$ & $3.4 \pm 2.2$ & $6.1 \pm 0.7$ & $4.9 \pm 1.2$ \\
\hline
\end{tabular}

1. $\bar{x} \pm$ ems. 2. Significant difference with control group $(\mathrm{p}<0.05)$

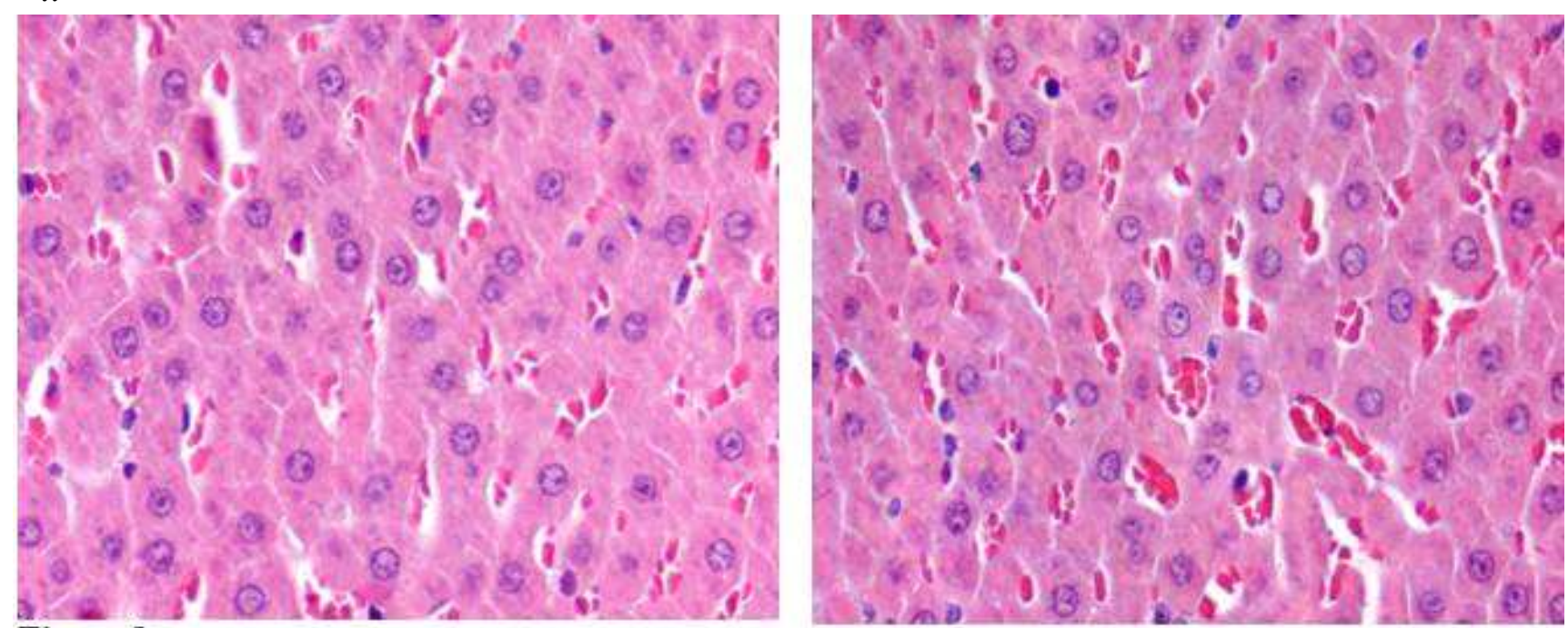

Figure 1. a. Liver. Acute study, animal control. Note normal architecture: form of hepatocytes and their nucleus. Staining H.E. 400X approx. b. Liver. Acute study, high-dosage animal. Note normal architecture and morphology of hepatocytes. Staining H.E. 400X approx

Pathology. There were 13 deaths during the subchronic study (eight rats within the first 16 days, one rat on days 44,48 , and 86 , respectively, and two rats on day 90). The deaths occurred in a female rat from the control group, and two females and two males from each treated group. The mortality rate was low and similar in all groups. At necropsy, tracheitis was observed in all cases.

Depletion of T areas was seen in spleen (higher in the lower dosage group) as the only significant finding (Figures $2 \mathrm{a}$ y $2 \mathrm{~b}$ ). The following findings were not significant: Kupffer cell activation, mild bile duct hyperplasia, megalocytosis in the liver (Figures 2c-2f), and hemoglobin pigment in the spleen (hematoxylineosin and Perl's stain, Figures $2 \mathrm{~g}$ and $2 \mathrm{~h}$ ).

\section{DISCUSSION}

The ethanol extract from Valeriana pavonii could be classified according to the Globally Harmonized Classification System for Chemical Substances and Mixtures (GHS) ${ }^{12}$ under the fifth category, taking into 
Figure 2. a. Spleen. Subchronic study. Animal control. Lymphoid follicle is seen at the germinal center $B(B)$ and the marginal zone or area $T(T)$. Staining H.E.100X approx. b. Spleen. Subchronic study. Lowdosage animal. Note the depletion of the T areas (T) compared with control. B areas may also be depleted (B). Staining H.E. 100X approx. c. Liver. Subchronic study. Animal control. Note the normal architecture of hepatocytes, their cytoplasm, nuclei and the normal population of Kupffer cells (arrow); Staining HE 400X approx. d. Liver. Subchronic study. High-dosage animal. Note the increase in the number of Kupffer cells (arrows); Staining HE400X approx. e. Liver. Subchronic study. Highdosage animal. Note bile duct hyperplasia (arrows); Figure 2c

Staining HE200X approx. f. Liver. Subchronic study. High-dosage animal. There are some enlarged nuclei (arrows) compared with normal (arrowhead). Staining H.E.400X approx. g. Spleen. Subchronic study. High-dosage animal. Intracellular brown pigments are evident (arrows); Staining HE200X approx. h. Spleen: subchronic study. High-dosage animal. Perl's staining for iron confirmed that spleen pigments are iron pigment derived from hemoglobin. (Arrows). Iron. Perl's stain 200X approx.

Figure 2a.
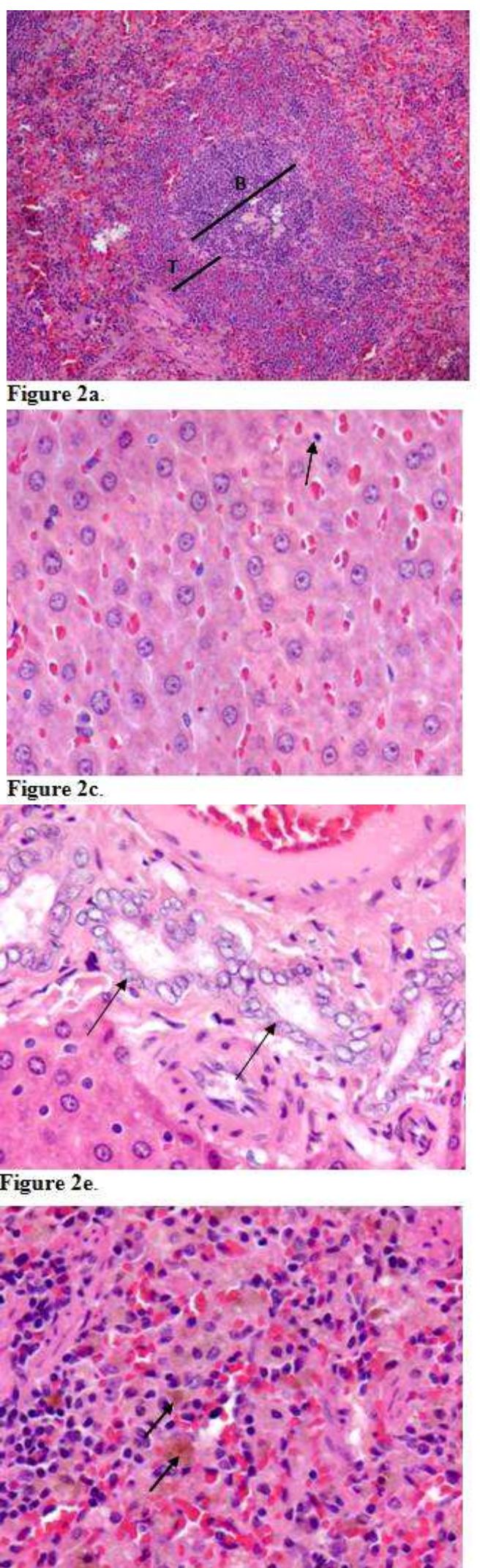

Figure 2g.

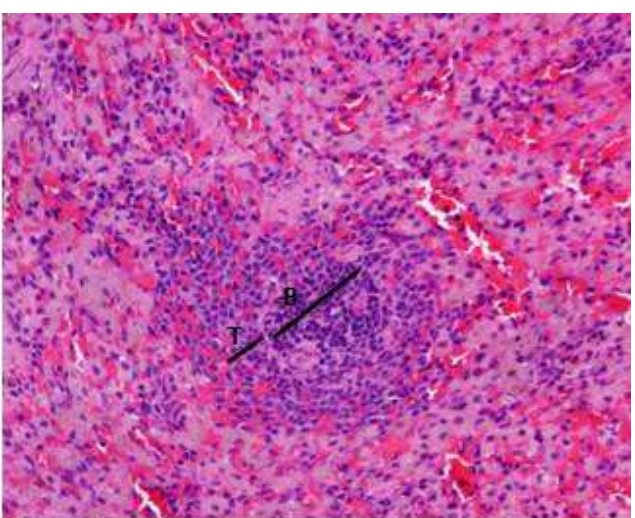

Figure 2b
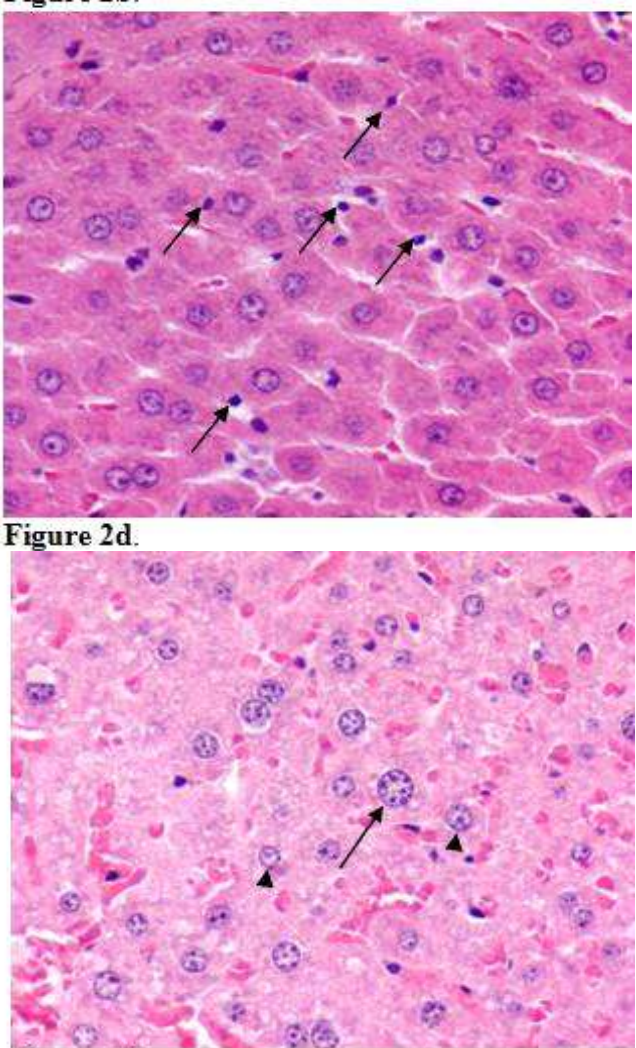

Figure 2f

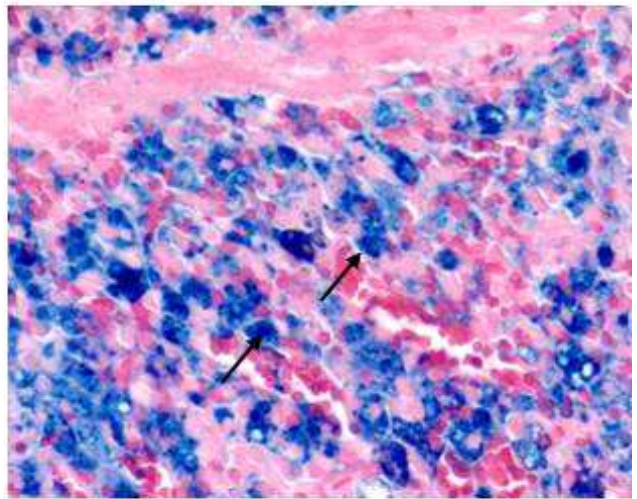

Figure 2h. 
account that the administration of this extract in a single $2000-\mathrm{mg} / \mathrm{kg}$ dose produced no deaths or signs of toxicity. While the acute and subchronic toxicity studies revealed no weight loss of the rodents. This finding was important because if an animal were to have lost weight, it would have been indicative of an illness process ${ }^{13}$.

In these studies, passivity seems to respond to sedative effects of $V$. pavonii, which have been previously evaluated ${ }^{6}$. These effects have already been reported for other Valeriana species, e.g., $V$. officinalis ${ }^{14,15}$. This parameter was marked in the subchronic toxicity study in which a dosage-response relationship was observed.

Throughout the acute study, it was observed that piloerection augmented slightly with time. This reaction could be because of the animal's level of stress increasing due to the loss of the $V$. pavonii sedative effects considering that the administration was done in a single dosage ${ }^{16}$. In the subchronic study, this reaction appeared during the first three weeks and it could be attributed to the stress produced by the daily gavage administration. This effect decreased gradually as a result of habituation and, possibly the anxiolytic effect of the extract ${ }^{6}$.

Aggressiveness and fear decreased over time because the degree of distress caused by the procedures was minimized given that animals are creatures of habit ${ }^{17}$. Although rats are not typically aggressive ${ }^{13}$, they showed a dosage-response effect with the administration of $V$. pavonii, suggesting that this species exerts sedative/ anxiolytic effects ${ }^{6}$.

The alarm reaction decreased over time. This seems to be related to the sedative effects of $V$. pavonii found in other species such as $V$. officinalis ${ }^{14,15}$. The sedative action of Valeriana has been attributed to the presence of several compounds like iridoids (valepotriates), decomposition products (baldrinals), and various components of the essential oil; particularly, the valerenic acid derivatives ${ }^{15}$. Furthermore, nasal discharge and dyspnea can be attributed to complications associated with gavage administration such as aspiration and airway injury ${ }^{16}$.

In the acute toxicity test, female rats showed statistically significant increases in serum AST levels, but there were no histopathological findings. Moreover, AST is not specific to liver function in rats and has low diagnostic value in this species ${ }^{13}$. The mild hyponatremia could be due to a mild hypotonic dehydration, while the direct bilirubin increase could be because it was not removed quickly from the serum and it could lead to its accumulation. Therefore, this finding will become significantif it is accompanied by total hyperbilirubinemia, physical findings, and results from other laboratory tests ${ }^{18}$.

The decrease in erythrocyte count in the highdosage group was compared with the hemoglobin and hematocrit normal values, suggesting anemia by hemodilution. This effect is frequently observed at high doses in toxicology studies with rats and it is generally considered to lack toxicological significance ${ }^{16}$.

Females and males presented differences in BUN, ALT, and AST serum levels. This could be due to variability associated with gender, strain, age, site of collection, anesthetic agents, among others ${ }^{13,16,19}$. Because of all these variations, it is important to compare with control groups from each study and include both genders in the study ${ }^{16,20}$.

In the histopathological study, the depletion of $\mathrm{T}$ areas in the spleen could not be attributed to the extract since this is a nonspecific reaction to stress, immunesuppression or to agonal changes ${ }^{21}$.

Kupffer cells located in the liver are targeted by toxic agents. They are not uncommon in toxicology studies and they show high variability ${ }^{16}$ but they are not considered as specific findings of toxicity. In bile ducts, slight hyperplasia may be physiological due to the increase in metabolic demand caused by the daily administration of a xenobiotic ${ }^{22}$.

Megalocytosis is commonly found in rats under physiological conditions. Moreover, in this study there were no alterations in liver enzyme levels and there were no other histopathological findings; hence, it could not be considered a finding of specific toxicity. The presence of pigments derived from hemoglobin in the spleen was normal, considering that many of the red blood cells destroyed were phagocytosed by reticuloendothelial cells ${ }^{23}$.

No relationship was found between the altered values of clinical chemistry (AST, sodium, direct bilirubin) and hematology (erythrocyte count), with histopathological findings. In addition, most lesions in the pathology and alterations in the blood parameters were presented in both treated and control groups. The deaths that occurred during the study were related to gastric gavage manipulation, leading first to fibrino-suppurative tracheitis 
and then death.

Although there were no signs of acute and subchronic toxicity attributable to the ethanolic extract of Valeriana pavonii, in $V$. officinalis, hepatotoxic reactions have been reported with valerian products; these reactions could be idiosyncratic ${ }^{14,24}$. There is no reported data on toxicity for long-term use of Valeriana. Toxicological studies have reported for the ethanol extract of $V$. officinalis an intraperitoneal $\mathrm{LD}_{50}$ of $3.3 \mathrm{mg} / \mathrm{kg}$ in rats $^{14}$, while the $\mathrm{LD}_{50}$ for this study was higher than $2000 \mathrm{mg} / \mathrm{kg}$. These values are not comparable because the route of administration was different. Through in vitro studies, it has been demonstrated that valepotriates (valtrate and didrovaltrate) have significant cytotoxic and mutagenic activity. However, toxicity was not demonstrated in vivo or in human clinical trials ${ }^{25}$. Therefore, it is important to complement this study with toxicity tests in vivo and in vitro to discard cytotoxicity and mutagenicity effects, keeping in mind that iridoids have been found in this species.

In conclusion, in acute and subchronic toxicity studies we did not observe mortality or signs of toxicity attributable to the administration of ethanol extract from $V$. pavonii and no significant weight loss was registered. Therefore, the NOAEL for the acute toxicity study was $2000 \mathrm{mg} / \mathrm{kg}$ and for the subchronic toxicity study was $1000 \mathrm{mg} / \mathrm{kg}$ weight. According to the dosage levels evaluated in the subchronic and acute toxicity studies, the LOAEL (Lowest Observed Adverse Effect Level) was not found for the $V$. pavonii ethanol extract.

Conflict of interest. None of the authors has conflicts of interest related to this study.

\section{ACKNOWLEDGEMENTS}

This study was funded by the Research Direction of the Bogotá campus, Research Vice-Presidency of Universidad Nacional de Colombia (Project DIB code: 8009046). The authors would like to thank the Department of Pharmacy, the Faculty of Sciences, and the Department of Toxicology, Faculty of Medicine, Universidad Nacional de Colombia.

\section{REFERENCES}

1. World Health Organization. WHO guidelines on safety monitoring of herbalmedicines in pharmacovigilance systems.
Geneva: WHO; 2004.

2. Bermúdez A, Oliveira-Miranda MA, Velázquez D. La investigación etnobotánica sobre plantas medicinales: Una revisión de sus objetivos y enfoques actuales. Interciencia. 2005; 30: 453-9.

3. Organización Mundial de la Salud. Estrategia de la OMS sobre medicina tradicional 2002-2005. Ginebra: OMS; 2002.

4. Beyra A, León MC, Iglesias E, Ferrándiz D, Herrera R, Volpato $\mathrm{G}$, et al. Estudios etnobotánicos sobre plantas medicinales en la provincia de Camagüey (Cuba). An Jard Bot Madr. 2004; 61: 185-204.

5. De Smet PA. Herbal remedies. NEngl J Med. 2002; 347: 204656.

6. Celis CT, Rincón J, Guerrero MF. Actividad farmacológica sobre el sistema nervioso central del extracto etanólico y de la fracción alcaloidal de V. pavonii. Rev Colomb Cien Quim Farm. 2007; 36: 11-22.

7. Giraldo SE, Rincón J, Puebla P, Marder M, Wasowski C, Vergel $\mathrm{N}$, et al. Isovaleramida: principio anticonvulsivante aislado de Valeriana pavonii. Biomedica. 2010; 30: 245-50.

8. Ministerio de la Protección Social, Instituto Nacional de Medicamentos y Alimentos. Normas farmacológicas 2006. Bogotá, DC: Ministerio de la Protección Social; 2006.

9. Ministerio de la Protección Social. Vademecum colombiano de plantas medicinales 2008. Bogotá, DC: Ministerio de la Protección Social; 2008.

10. Moser VC, Cheek BM, McPhail RC. A multidisciplinary approach to toxicological screening: III. Neurobehavioral toxicity. J Toxicol Environ Health A. 1995; 45: 173-210.

11. Twish JWR.Applied longitudinal data analisisfor epidemiology: a practical guide. New York: Cambridge University Press; 2005.

12. Naciones Unidas. Sistema global armonizado de clasificación y etiquetado de productos químicos (SGA). Parte 3, Peligros para la salud. $2^{\mathrm{a}}$ ed. Ginebra: Naciones Unidas; 2007. p. 115.

13. Suckow M, La Regina M, Sharp P. The laboratory rat. Boca Raton: CRC Press; 2000.

14. Barnes J, Anderson L, Phillipson J. Plantas medicinales. Barcelona: Pharma Editores; 2004.

15. Marder M. Viola H, Wasowski C, Fernández S, Medina JH, Paladín AC. 6-methylapigenina and hesperidin: new valeriana flavonoids with activity on the CNS. Pharmacol Biochem Behav. 2003; 75: 537-45.

16. Suckow M., Weisbroth S, Franklin C. The laboratory rat. $2^{\text {nd }}$ ed. London: Elsevier Academic Press; 2006.

17. Bennett BT, Brown MJ, Schofield JC. Essentials for animal research a primerfor research personnel. Chapter 2, Alternative methodologies. p. 9. $2^{\text {nd }}$ ed. Maryland: USDA; 1994. [fecha de acceso 7 de mayo de 2006]. URL disponible en: http:// www.nal.usda.gov/awic/pubs/noawicpubs/essentia.htm\#2

18. Thrall M, Baker D, Lassen E, Campbell T, Rebar A, DeNicola $\mathrm{D}$, etal. Veterinary hematologyand clinical chemistry. Baltimore: Lippincott Williams \& Wilkins; 2004.

19. Reilly J. Variables in animal based research: Variability associated with experimental conditions and techniques. ANZCCART News. 1998; 11 Pt2: 1-7.

20. Petterino C, Argentino-Storino A. Clinical chemistry and 
haematology historical data in control Sprague-Dawley rats from pre-clinical toxicity studies. Exp Toxicol Pathol. 2006;57: 213-19.

21. Greave P. Histopathology of preclinical toxicity studies. $3^{\text {rd }} \mathrm{ed}$ London: Elsevier; 2007.

22. Grasso P. Essentials of pathology for toxicologists. London: Taylor \& Francis; 2002.
23. Guyton A, Hall J. Tratado de fisiología médica. $10^{a}$ ed. México: McGraw-Hill Interamericana; 2001

24. Stevinson C, Ernst E. Valerian for insomnia: a systematic review of randomized clinical trials. Sleep Med. 2000; 1: 91-9.

25. Yao M, Ritchie H, Brown P. A developmental toxicityscreening test of valerian. J Ethnopharmacol. 2007; 113: 2049 . 